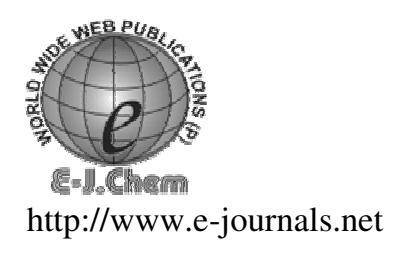

ISSN: 0973-4945; CODEN ECJHAO

E-Journal of Chemistry

Vol. 5, No., pp. 409-426, April 2008

\title{
Novel Activated Carbons from Agricultural Wastes and their Characterization
}

\author{
S.KARTHIKEYAN*, P.SIVAKUMAR and P.N.PALANISAMY \\ *Department of Chemistry, Erode Sengunthar Engineering College, Thudupathi, Erode. \\ Department of Chemistry, Kongu Engineering College, Perundurai, Erode, India.
} Tel :094422 64501; skmush@ rediffmail.com

Received 30 July 2007; Accepted 20 September 2007

\begin{abstract}
Solid waste disposal has become a major problem in India, Either it has to be disposed safely or used for the recovery of valuable materials as agricultural wastes like turmeric waste, ferronia shell waste, jatropha curcus seed shell waste, delonix shell waste and ipomea carnia stem. Therefore these wastes have been explored for the preparation of activated carbon employing various techniques. Activated carbons prepared from agricultural solid wastes by chemical activation processes shows excellent improvement in the surface characteristics. Their characterization studies such as bulk density, moisture content, ash content, fixed carbon content, matter soluble in water, matter soluble in acid, $\mathrm{pH}$, decolourising power, phenol number, ion exchange capacity, ion content and surface area have been carried out to assess the suitability of these carbons as absorbents in the water and wastewater. For anionic dyes (reactive, direct, acid) a close relationship between the surface area and surface chemical groups of the modified activated carbon and percentage of dye removal by adsorption can be observed. Cationic dyes large amount of surface chemical groups present in the sample (mainly carboxylic, anhydrides, lactones and phenols etc.) are good anchoring sites for adsorption. The present study reveals the recovery of valuable adsorbents from readily and cheaply available agriculture wastes.
\end{abstract}

Keywords : Turmeric waste, Ferronia shell, Jatropha curcus seed shell, Delonix shell, Ipomea Carnia Stem and Activated carbon.

\section{Introduction}

Comprising over two third of the Earth's surface, water is undoubtedly the most precious natural divine resource that exists in our planet. Although we, as humans, recognize this fact, we disregard it by polluting our rivers, lakes and oceans. Subsequently, we are slowly but surely harming our planet to the point where organisms will die at a very alarming rate. In addition to innocent organisms dieing off, our drinking water has also become greatly affected. In order to combat water pollution, we must understand the problems and become part of the solution. 
The use of charcoal extends far back into history. Ancient Hindus in India used it for drinking water filtration and Egyptians used carbonized wood as a medical adsorbent and purifying agent as early as 1500 B.C ${ }^{1}$.

Activated carbon was first introduced industrially in the first part of the $20^{\text {th }}$ century, when activated carbon from vegetable material was produced for use in sugar refining ${ }^{2}$. Powdered activated carbon was first produced commercially in Europe in the $19^{\text {th }}$ century, using wood as a raw material, which found wide use in the sugar industry. In the US, the first production of activated carbon used black ash as the source, after it was accidentally discovered that the ash was very effective in decolorizing liquids ${ }^{3}$. Activated carbon has since then been used extensively for this purpose in many industries. In particular, it has been commonly used for the removal of organic dyes from textile waste waster. Agricultural by products and waste materials used for the production of activated carbons include olive stones ${ }^{4}$, almond shells ${ }^{5}$, apricot and peach stones ${ }^{6}$, maize $\operatorname{cob}^{7}$, linseed straw, saw dust $^{8}$, rice hulls ${ }^{9}$, cashew nut hull, cashew nut sheath ${ }^{10}$, coconut shells and husks ${ }^{11}$, eucalyptus bark ${ }^{12}$, linseed cake, tea waste $\mathrm{ash}^{13}$. Besides these, other sources of activated carbon are sulfonated coall ${ }^{14}$, tyre coal dust ${ }^{15}$, activated bauxite, cement kiln dust ${ }^{16}$, shale oil ash $^{17}$, ground sunflower stalk ${ }^{18}$ etc. All these activated carbons have been successfully used for the adsorption processes.

Therefore, carbons with excellent surface properties and specific functionalities should be developed to create a high affinity for adsorbate adsorption. It has been shown that total accessible surface area and pore volume for adsorption is inversely proportional to macromolecular size $\mathrm{e}^{21-23}$. It will be beneficial to have an activated carbon with a sufficient amount of supermicroporosity and mesoporosity for enhanced solute adsorption. Five Biomass materials, with different characteristics, were subjected to the following nine different treatments: Impregnation with zinc chloride, calcium chloride, sodium sulphate, sodium carbonate, calcium carbonate (Dolomite Process), acid process with sulphuric acid, hydrochloric acid, sulphuric acid + ammonium per sulphate and sulphuric acid + hydrogen peroxide. These treatment methods were selected to: (1) produce a wide range of carbons with different surface chemistry and porosity and (2) elucidate the most suitable processes for development of the best materials for adsorbate uptake.

\section{Experimental}

Agricultural wastes were collected from in and around Erode City, Tamil Nadu, India. They were cut into small pieces ( $3 \mathrm{~cm}$ size app.), dried in sunlight until all the moisture was evaporated. The dried material was used for the preparation of activated carbons using physical and chemical activation methods.

\section{Carbonization procedures ${ }^{24}$}

\section{Carbonization with chloride salts}

The material to be carbonized is impregnated with solution of chloride salts such as zinc chloride, calcium chloride for $24 \mathrm{~h}$. Accordingly sufficient quantities were soaked well with $10 \%$ chloride solution of 5 liters capacity respectively so that the solution get well adsorbed for a period of 24 hours. At the end of 24 hours the excess chloride solution were decanted off and air-dried. Then the materials were placed in muffle furnace carbonized at $400{ }^{\circ} \mathrm{C}$. The dried materials were powdered and activated in a muffle furnace kept at $800{ }^{\circ} \mathrm{C}$ for a period of 10 minutes. After the activation, the carbon washed sufficiently with $4 \mathrm{~N} \mathrm{HCl}$ to remove the cations. Then the materials were washed with plenty of water to remove excess acid, dried and powdered. 


\section{Carbonization with sulphate salts}

In this method the agricultural wastes to be carbonized were soaked in $10 \%$ solutions of sodium sulphate for a period of 24 hours. After impregnation, the liquid portion was decanted off and then dried. The dried mass was subjected to carbonization process at $400{ }^{\circ} \mathrm{C}$ powdered well and finally thermally activated at $800{ }^{\circ} \mathrm{C}$ for a period of 10 minutes.

\section{Carbonization with carbonate salts}

The materials to be carbonized were soaked with $10 \%$ sodium carbonate solution for a period of 24 hours. After impregnation, the liquid portion was decanted off and the material dried. The dried mass was subjected to carbonization process at $400{ }^{\circ} \mathrm{C}$, powdered well and finally activated at a temperature of $800{ }^{\circ} \mathrm{C}$ for a period of 10 minutes.

\section{Dolomite process}

Sufficient quantities of dried Agricultural wastes were taken over a calcium carbonate bed and the upper layer of waste was also covered with a layer of calcium carbonate. The whole material was carbonized at $400{ }^{\circ} \mathrm{C}$, powdered well and followed by the thermal activation at $800{ }^{\circ} \mathrm{C}$. After the activation, the material was repeatedly washed with plenty of water to remove calcium carbonate and dried $110{ }^{\circ} \mathrm{C}$.

\section{Acid process}

The dried material was treated with excess of sulphuric acid and hydrochloric acid. charring of the occurred immediately, accompanied by evolution of heat and fumes. When the reaction subsided, the mixture was left in an air oven maintained at $140-160{ }^{\circ} \mathrm{C}$ for a period of 24 hours. At the end of this period, the product was washed with large volume of water to remove free acid, dried at $110^{\circ} \mathrm{C}$ and finally activated at $800^{\circ} \mathrm{C}$.

\section{Chemical activation with $\mathrm{H}_{2} \mathrm{SO}_{4}$ and $\mathrm{H}_{2} \mathrm{SO}_{4}+\mathrm{NH}_{4} \mathrm{~S}_{2} \mathrm{O}_{8}$}

In this method 1 part of the material and 1.5 parts of $\mathrm{H}_{2} \mathrm{SO}_{4}$ were mixed with 0.4 parts of $\mathrm{NH}_{4} \mathrm{~S}_{2} \mathrm{O}_{8}$ and kept in muffle furnace at $120^{\circ} \mathrm{C}$ for 12 hours. At the end of this period, the product was washed with large volume of water to remove free acid, dried at $110{ }^{\circ} \mathrm{C}$ and finally activated at $800^{\circ} \mathrm{C}$.

\section{Characterization of the carbons}

$\mathrm{pH}$ and conductivity were analyzed using Elico make $\mathrm{pH}$ meter (model L1-120) and conductivity meter (model M-180) respectively. Moisture content (\%) by mass, ash (on dry basis) $\%$ by mass, volatile matter, fixed carbon content, bulk density, specific gravity, porosity, meter soluble in water, matter soluble in acid, phenol adsorption capacity, carbon tetrachloride activity, iron content and iodine number were analyzed as per standard procedures $^{25}$. Estimation of sodium, potassium were done using Systronics make Flame Photometer. The BET surface area was measured using Quantachrome analyzer.

\section{Dye Adsorption Studies}

In this part of study $0.1 \mathrm{~g}$ of adsorbent agitated with $50 \mathrm{~mL}$ of $50 \mathrm{ppm}$ dye solution for a period of $2 \mathrm{~h}$. Finally the supernatant solution filtered using whatman filter paper No. 41 and analysed spectrophotometrically using Elico U.V.Visible Spectrometer (Model : BL 192) for residual dye concentration. 


\section{Results and Discussion}

\section{Turmeric Waste Activated Carbon}

The evolution of characteristics of nine types of activated carbon derived from turmeric waste indicates that the carbon obtained by acid process has higher bulk density than the carbon prepared by other process. It was observed that carbon prepared by $\mathrm{ZnCl}_{2}$ impregnation process has lower bulk density. As reported, the observed decrease in the bulk density may be due to increase in the pore system with $\mathrm{ZnCl}_{2}$ impregnation ${ }^{10}$.

From Table 1, the moisture content was found to be higher in case of carbons obtained by $\mathrm{ZnCl}_{2}$ and $\mathrm{CaCl}_{2}$ process. Even though moisture content of the carbon has no effect on its adsorptive power, it dilutes the carbon which necessitates the use of additional weight of carbon during treatment process.

Among the carbons prepared by various methods, the carbon obtained by dolomite process was found to contain less moisture compared to the carbon obtained by chloride process. Ash content generally gives an idea about inorganic constituents associated with carbon obtained by different carbonization methods. The ash content values from Table 1 indicate that the overall ash content for all the varieties of carbon prepared shows comparable lesser values. This may be attributed to lower inorganic content and higher fixed carbon.

Solubility studies of carbon in acid and water were performed to evaluate the amount of impurities present in the carbon prepared by different carbonization process. The solubility studies were preformed since the presence of impurities in the carbon may affect the expected quality of the treated water during treatment. From Table 1 the data pertaining to the matter soluble in water and in acid indicates that, except carbon obtained by acid process, the carbon prepared by all other processes exhibits moderate level of impurities. The high value of water soluble matter is prepared by carbonate process which indicates that a large amount of carbonate salts would have been incorporated into the carbon structure. The acid leachable matter was also found to be higher in the carbon prepared by the carbonate process.

The carbon derived in acid process is acidic in nature with moderate conductivity compared with the carbon prepared by other carbonization process. As reported by Bansal R.C et $a l .^{26}$, this may be due to the incorporation of acid in the carbon structure. Chloride impregnated carbon is also found with moderate conductivity. This may be due to the development of exchangeable sites on the surface of the activated carbon.

The iron content is considerably lower in all the varieties of carbon prepared by different methods. This implies that the carbon prepared by different carbonization process can be utilized in the treatment of effluent water without the problem of iron leaching into the treated water. The level of sodium and potassium was found to be moderate and slightly high in the carbons prepared by chloride process. This can be taken as an advantage for ion exchange process, the surface area of carbons prepared in all methods found to be in the following order.

$$
\mathrm{ZnCl}_{2}>\mathrm{CaCl}_{2}>\mathrm{H}_{2} \mathrm{SO}_{4}+\mathrm{NH}_{4} \mathrm{~S}_{2} \mathrm{O}_{8}>\mathrm{HCl}>\mathrm{H}_{2} \mathrm{SO}_{4}+\mathrm{H}_{2} \mathrm{O}_{2}>\mathrm{H}_{2} \mathrm{SO}_{4}>\mathrm{Na}_{2} \mathrm{SO}_{4}>
$$
$\mathrm{Na}_{2} \mathrm{CO}_{3}>\mathrm{CaCO}_{3}$ (Dolomite)

The higher surface area of carbon prepared by chloride process $\left(\mathrm{ZnCl}_{2} \& \mathrm{CaCl}_{2}\right)$ may be due to effective removal of organic matter by chloride process. Table 1 shows that the surface area of activated carbon prepared from turmeric waste are comparable with the other activated carbons prepared from low cost materials. The phenol adsorption capacity and $\mathrm{CCl}_{4}$ activity is found in the same order as that of surface area. This indicates that the carbon prepared by chloride process has the maximum surface area, carbons with high surface area are considered most suitable for adsorption of adsorbates in aqueous solutions. 
Table 1. Turmeric waste activated carbon properties

\begin{tabular}{|c|c|c|c|c|c|c|c|c|c|}
\hline Properties & $\mathrm{HCl}$ & $\mathrm{H}_{2} \mathrm{SO}_{4}$ & $\mathrm{ZnCl}_{2}$ & $\mathrm{Na}_{2} \mathrm{SO}_{4}$ & $\mathrm{Na}_{2} \mathrm{CO}_{3}$ & $\mathrm{CaCO}_{3}$ & $\mathrm{CaCl}_{2}$ & $\begin{array}{r}\mathrm{H}_{2} \mathrm{SO}_{4}+ \\
\mathrm{NH}_{4} \mathrm{~S}_{2} \mathrm{O}_{8} \\
\end{array}$ & $\begin{array}{c}\mathrm{H}_{2} \mathrm{SO}_{4} \\
+ \\
\mathrm{H}_{2} \mathrm{O}_{2} \\
\end{array}$ \\
\hline $\mathrm{pH}$ & 8.95 & 5.70 & 6.00 & 8.13 & 6.31 & 9.63 & 7.69 & 6.80 & 7.08 \\
\hline $\begin{array}{l}\text { Moisture } \\
\text { Content, \% }\end{array}$ & 15.80 & 16.20 & 28.80 & 9.80 & 11.20 & 6.40 & 17.20 & 11.80 & 7.80 \\
\hline Ash content $\%$ & 10.68 & 14.46 & 10.16 & 13.89 & 10.69 & 8.78 & 14.20 & 8.36 & 8.86 \\
\hline $\begin{array}{l}\text { Volatile } \\
\text { Matter, \% }\end{array}$ & 15.40 & 10.30 & 11.40 & 17.10 & 14.20 & 18.40 & 14.00 & 7.20 & 9.10 \\
\hline Fixed Carbon \% & 68.8 & 73.5 & 59.8 & 73.1 & 74.6 & 75.2 & 68.8 & 81.0 & 83.1 \\
\hline $\begin{array}{l}\text { Conductivity, } \\
\mathrm{mS} / \mathrm{cm}\end{array}$ & 0.22 & 0.10 & 0.61 & 0.13 & 0.33 & 0.37 & 0.24 & 0.19 & 0.42 \\
\hline $\begin{array}{l}\text { Specific } \\
\text { gravity, }(\mathrm{S})\end{array}$ & 1.25 & 1.33 & 1.49 & 0.89 & 1.10 & 1.48 & 1.32 & 1.88 & 1.37 \\
\hline $\begin{array}{l}\text { Bulk density } \\
\text { (D) }\end{array}$ & 0.76 & 0.89 & 0.41 & 0.44 & 0.83 & 0.52 & 0.46 & 0.86 & 0.79 \\
\hline $\begin{array}{l}\text { Porosity } \\
(\mathrm{S}-\mathrm{D} / \mathrm{S}) 100, \%\end{array}$ & 39.20 & 33.08 & 72.48 & 50.56 & 24.55 & 64.86 & 65.15 & 54.26 & 42.34 \\
\hline $\begin{array}{l}\text { Matter Soluble } \\
\text { in water, } \%\end{array}$ & 1.42 & 0.48 & 1.78 & 2.03 & 0.90 & 2.88 & 2.06 & 0.81 & 0.84 \\
\hline $\begin{array}{l}\text { Matter Soluble } \\
\text { in Acid, } \%\end{array}$ & 1.44 & 0.23 & 1.12 & 1.50 & 0.91 & 1.70 & 1.43 & 0.81 & 0.44 \\
\hline $\begin{array}{l}\text { Surface Area, } \\
\mathrm{m}^{2} / \mathrm{g}\end{array}$ & 790 & 361 & 1350 & 352 & 291 & 221 & 1264 & 898 & 703 \\
\hline $\begin{array}{l}\text { Iodine Number } \\
\mathrm{mg} / \mathrm{g}\end{array}$ & 755 & 342 & 1265 & 332 & 282 & 212 & 1210 & 839 & 676 \\
\hline $\begin{array}{l}\text { Sodium, } \\
\text { W / W \% }\end{array}$ & 6.40 & 5.50 & 8.40 & 5.40 & 2.10 & 9.00 & 15.00 & 5.50 & 8.00 \\
\hline $\begin{array}{l}\text { Potassium } \\
\text { W / W \% }\end{array}$ & 7.60 & 7.10 & 10.60 & 5.70 & 3.10 & 6.10 & 2.00 & 5.00 & 5.00 \\
\hline $\begin{array}{l}\text { Iron Content } \\
\mathrm{W} / \mathrm{W} \%\end{array}$ & 0.80 & 1.00 & 1.20 & 1.40 & 1.20 & 1.00 & 0.90 & 1.10 & 1.20 \\
\hline $\mathrm{CCl}_{4}$ Activity & 0.52 & 0.72 & 1.32 & 0.36 & 0.55 & 0.59 & 1.19 & 1.02 & 1.14 \\
\hline $\begin{array}{l}\text { Phenol } \\
\text { Adsorption } \\
\text { Capacity, mg /g }\end{array}$ & 3.20 & 2.50 & 5.80 & 1.40 & 3.20 & 0.80 & 4.20 & 3.00 & 2.50 \\
\hline Yield, \% & 32 & 55 & 42 & 39 & 44 & 62 & 37 & 52 & 55 \\
\hline
\end{tabular}


From Table 1 it is clear that Dolomite process gives more yields followed by acid process when compared to other treatment process, in the acid processes $\mathrm{H}_{2} \mathrm{SO}_{4}$ gives high yield, due to its high charring capacity.

\section{Jatropha Curcus Seed Shell Waste Activated Carbon}

Jatropha curcus will find a prime importance during the next decade. Bio-diesel prepared from jatropha seed will definitely help in preserving the non renewable energy resources. During the extraction of Bio diesel from jatropha seed, the shell disposal is going to be a concern for environ mentalists. The objective of aiming activated carbon preparation using jatropha seed shell will reduce the burden environmental pollution.

Table 2 shows that the bulk density values are on the lower side, which may be due to highly branched and porous carbon with more void space. Except $\mathrm{H}_{2} \mathrm{SO}_{4}, \mathrm{ZnCl}_{2}, \mathrm{Na}_{2} \mathrm{CO}_{3}$ and $\mathrm{NH}_{4} \mathrm{~S}_{2} \mathrm{O}_{8}$ carbon all the carbons found to be basic in nature.

Moisture content was found low only in $\mathrm{H}_{2} \mathrm{SO}_{4}$ carbon even though it has comparable surface area. $\mathrm{HCl}$ and $\mathrm{NH}_{4} \mathrm{~S}_{2} \mathrm{O}_{8}$ carbons contains very little amount of ash content, which might be an indication of maximum removal of inorganic content. Due to the presence of highly porous organic matter in jatropha shell, the quantity of volatile matter shows a higher trend. High value of ash and volatile matter reduces the quantity of fixed carbon.

Due to the presence of both $+v e$ and $-v e$ ions in the porous areas results in an increased conductivity. Matter soluble in water and acid did not give any conclusion on the effect of process conditions on the removal of impurities. Lower bulk density enhances the trapping of impurities inside the porous structure. Sodium and potassium content may be due to the presence of mineral $\mathrm{Na}$ and $\mathrm{K}$ in the jatropha seed shell.

Iron content is constant for almost all the processes and did not give any conclusion about the effect of process condition on the iron content. This quantity will not be sufficient to cause any adverse effects during water treatment by leaching. Except dolomite process all the remaining eight processes show encouraging results on making more pores to attain high surface area to improve adsorptive capacity. Linear relationship of $\mathrm{CCl}_{4}$ activity, iodine number and phenol adsorption capacity with surface area further confirms the result of surface area.

The order of surface area of carbons prepared with different process given as follows. $\mathrm{HCl}>\mathrm{CaCl}_{2}>\mathrm{ZnCl}_{2}>\mathrm{H}_{2} \mathrm{SO}_{4}+\mathrm{H}_{2} \mathrm{O}_{2}>\mathrm{H}_{2} \mathrm{SO}_{4}>\mathrm{H}_{2} \mathrm{SO}_{4}+\mathrm{NH}_{4} \mathrm{~S}_{2} \mathrm{O}_{8}>\mathrm{Na}_{2} \mathrm{CO}_{3}>\mathrm{Na}_{2} \mathrm{SO}_{4}>$ Dolomite

In all the processes the final carbon yield found nearly $40 \%$ only. Only $\mathrm{H}_{2} \mathrm{SO}_{4}+\mathrm{NH}_{4}$ $\mathrm{S}_{2} \mathrm{O}_{8}$ process gives an encouraging result on yield of $62 \%$.

\section{Delonix Regia Seed Shell Waste Activated Carbon}

The characteristics of carbon prepared from delonix regia seed shell waste by different carbonization process are given in the Table 3. The data indicates that the carbon obtained by $\mathrm{Na}_{2} \mathrm{CO}_{3}$ process show higher bulk density when compared to other process. Carbon prepared by dolomite and $\mathrm{ZnCl}_{2}$ process has lower bulk density. This lower bulk density of carbon may be due to high spongy nature of the material.

On analyzing the moisture content of all the activated carbons, the carbon prepared by $\mathrm{HCl}$ process has high moisture content. As discussed earlier this is due to adsorption of moisture from atmosphere by highly porous carbon. In the same manner lower moisture content is noticed less porous carbons due to its poor adsorptive capacity. 
Table 2 Jatropha curcus seed shell waste activated carbon properties

\begin{tabular}{|c|c|c|c|c|c|c|c|c|c|}
\hline Properties & $\mathrm{HCl}$ & $\mathrm{H}_{2} \mathrm{SO}_{4}$ & $\mathrm{ZnCl}_{2}$ & $\mathrm{Na}_{2} \mathrm{SO}_{4}$ & $\mathrm{Na}_{2} \mathrm{CO}_{3}$ & $\mathrm{CaCO}_{3}$ & $\mathrm{CaCl}_{2}$ & $\begin{array}{l}\mathrm{H}_{2} \mathrm{SO}_{4}+ \\
\mathrm{NH}_{4} \mathrm{~S}_{2} \mathrm{O}_{8}\end{array}$ & $\begin{array}{r}\mathrm{H}_{2} \mathrm{SO}_{4} \\
+\mathrm{H}_{2} \mathrm{O}_{2} \\
\end{array}$ \\
\hline $\mathrm{pH}$ & 9.55 & 4.67 & 6.60 & 8.50 & 5.74 & 9.20 & 7.16 & 6.85 & 7.05 \\
\hline $\begin{array}{l}\text { Moisture } \\
\text { content, } \%\end{array}$ & 11.00 & 2.00 & 8.00 & 10.00 & 10.00 & 16.40 & 8.20 & 9.60 & 10.10 \\
\hline $\begin{array}{l}\text { Ash content, } \\
\%\end{array}$ & 7.42 & 11.94 & 19.57 & 17.77 & 14.00 & 10.57 & 13.07 & 8.67 & 10.85 \\
\hline $\begin{array}{l}\text { Volatile } \\
\text { matter \% }\end{array}$ & 30.00 & 26.70 & 27.50 & 31.20 & 20.00 & 24.20 & 27.80 & 31.30 & 34.20 \\
\hline $\begin{array}{l}\text { Fixed } \\
\text { carbon, } \%\end{array}$ & 69.00 & 73.50 & 59.80 & 73.10 & 73.80 & 72.10 & 69.70 & 81.00 & 84.30 \\
\hline $\begin{array}{l}\text { Conductivity } \\
\mathrm{mS} / \mathrm{cm}\end{array}$ & 0.22 & 0.10 & 0.61 & 0.13 & 0.33 & 0.37 & 0.24 & 0.19 & 0.42 \\
\hline $\begin{array}{l}\text { Specific } \\
\text { gravity, S }\end{array}$ & 1.25 & 1.33 & 1.49 & 0.89 & 1.10 & 1.48 & 1.32 & 1.88 & 1.37 \\
\hline $\begin{array}{l}\text { Bulk } \\
\text { density, D }\end{array}$ & 0.44 & 0.39 & 0.44 & 0.40 & 0.63 & 0.29 & 0.39 & 0.28 & 0.45 \\
\hline $\begin{array}{l}\text { Porosity } \\
(\mathrm{S}-\mathrm{D} / \mathrm{S}) \\
\mathrm{X} 100, \%\end{array}$ & 44.20 & 53.08 & 69.48 & 62.56 & 64.55 & 64.86 & 72.15 & 61.26 & 72.34 \\
\hline $\begin{array}{l}\text { Matter } \\
\text { soluble in } \\
\text { water, \% }\end{array}$ & 1.84 & 1.06 & 1.74 & 2.12 & 1.41 & 1.22 & 0.72 & 1.02 & 1.20 \\
\hline $\begin{array}{l}\text { Matter } \\
\text { soluble in } \\
\text { Acid, \% }\end{array}$ & 1.25 & 1.08 & 1.62 & 1.42 & 0.94 & 0.74 & 0.92 & 1.41 & 1.91 \\
\hline $\begin{array}{l}\text { Surface } \\
\text { area, } \mathrm{m}^{2} / \mathrm{g}\end{array}$ & 1195 & 709 & 950 & 456 & 629 & 408 & 1064 & 664 & 751 \\
\hline $\begin{array}{l}\text { Iodine } \\
\text { number, } \\
\mathrm{mg} / \mathrm{g}\end{array}$ & 1143 & 668 & 899 & 412 & 589 & 368 & 998 & 612 & 723 \\
\hline $\begin{array}{l}\text { Sodium } \\
\text { W / W \% }\end{array}$ & 15.00 & 5.50 & 8.00 & 15.00 & 5.50 & 8.00 & 6.40 & 5.50 & 8.40 \\
\hline $\begin{array}{l}\text { Potassium, } \\
\text { W / W \% }\end{array}$ & 10.60 & 5.70 & 3.10 & 6.10 & 2.00 & 5.00 & 5.00 & 7.60 & 7.10 \\
\hline $\begin{array}{l}\text { Iron Content, } \\
\mathrm{W} / \mathrm{W} \%\end{array}$ & 1.40 & 1.20 & 1.00 & 0.90 & 1.10 & 1.20 & 0.80 & 1.00 & 1.20 \\
\hline $\begin{array}{l}\mathrm{CCl}_{4} \\
\text { Activity }\end{array}$ & 0.36 & 0.55 & 0.59 & 1.14 & 1.19 & 0.52 & 0.72 & 1.32 & 1.19 \\
\hline $\begin{array}{l}\text { Phenol } \\
\text { adsorption } \\
\text { capacity } \\
\mathrm{mg} / \mathrm{g}\end{array}$ & 5.21 & 2.85 & 3.62 & 1.22 & 2.28 & 0.80 & 4.20 & 3.08 & 2.64 \\
\hline Yield, \% & 42 & 39 & 44 & 32 & 55 & 37 & 52 & 62 & 32 \\
\hline
\end{tabular}


Table 3 Delonix regia seed shell waste activated carbon properties

\begin{tabular}{|c|c|c|c|c|c|c|c|c|c|}
\hline Properties & $\mathrm{HCl}$ & $\mathrm{H}_{2} \mathrm{SO}_{4}$ & $\mathrm{ZnCl}_{2}$ & $\mathrm{Na}_{2} \mathrm{SO}_{4}$ & $\mathrm{Na}_{2} \mathrm{CO}_{3}$ & $\mathrm{CaCO}_{3}$ & $\mathrm{CaCl}_{2}$ & $\begin{array}{l}\mathrm{H}_{2} \mathrm{SO}_{4}+ \\
\mathrm{NH}_{4} \mathrm{~S}_{2} \mathrm{O}_{8} \\
\end{array}$ & $\begin{array}{r}\mathrm{H}_{2} \mathrm{SO}_{4} \\
+\mathrm{H}_{2} \mathrm{O}_{2} \\
\end{array}$ \\
\hline $\mathrm{pH}$ & 5.65 & 6.21 & 5.25 & 8.67 & 9.20 & 8.20 & 8.03 & 8.06 & 6.80 \\
\hline $\begin{array}{l}\text { Moisture } \\
\text { content, \% }\end{array}$ & 12.20 & 6.30 & 8.10 & 6.50 & 4.70 & 9.10 & 6.00 & 4.00 & 6.00 \\
\hline $\begin{array}{l}\text { Ash content, } \\
\%\end{array}$ & 10.64 & 10.64 & 1.96 & 12.09 & 17.42 & 17.02 & 17.27 & 13.67 & 10.83 \\
\hline $\begin{array}{l}\text { Volatile } \\
\text { matter \% }\end{array}$ & 18.50 & 24.20 & 29.00 & 24.80 & 21.30 & 27.20 & 20.20 & 19.50 & 21.40 \\
\hline $\begin{array}{l}\text { Fixed } \\
\text { carbon, \% }\end{array}$ & 69.3 & 69.5 & 62.9 & 68.7 & 74.0 & 63.7 & 73.8 & 76.5 & 72.6 \\
\hline $\begin{array}{l}\text { Conductivity } \\
\mathrm{mS} / \mathrm{cm}\end{array}$ & 0.13 & 0.29 & 0.19 & 0.17 & 0.05 & 0.24 & 0.14 & 0.16 & 0.08 \\
\hline $\begin{array}{l}\text { Specific } \\
\text { gravity, } S\end{array}$ & 1.17 & 0.97 & 0.98 & 0.96 & 1.20 & 1.19 & 1.07 & 0.92 & 1.02 \\
\hline $\begin{array}{l}\text { Bulk } \\
\text { density, D }\end{array}$ & 0.43 & 0.37 & 0.30 & 0.34 & 0.53 & 0.30 & 0.43 & 0.38 & 0.41 \\
\hline $\begin{array}{l}\text { Porosity } \\
\text { (S-D/S) } \\
\text { X100, \% }\end{array}$ & 63.25 & 61.86 & 69.39 & 64.58 & 55.83 & 74.79 & 59.81 & 58.70 & 59.80 \\
\hline $\begin{array}{l}\text { Matter } \\
\text { soluble in } \\
\text { water, \% }\end{array}$ & 0.85 & 0.51 & 0.87 & 0.43 & 1.00 & 1.68 & 0.55 & 0.25 & 0.85 \\
\hline $\begin{array}{l}\text { Matter } \\
\text { soluble in } \\
\text { Acid, \% }\end{array}$ & 0.63 & 0.48 & 0.43 & 0.45 & 0.62 & 1.80 & 0.68 & 0.45 & 0.85 \\
\hline $\begin{array}{l}\text { Surface } \\
\text { area, } \mathrm{m}^{2} / \mathrm{g}\end{array}$ & 1163 & 404 & 322 & 431 & 419 & 770 & 281 & 319 & 413 \\
\hline $\begin{array}{l}\text { Iodine } \\
\text { number, } \\
\mathrm{mg} / \mathrm{g}\end{array}$ & 1114 & 388 & 309 & 414 & 414 & 739 & 270 & 300 & 388 \\
\hline $\begin{array}{l}\text { Sodium } \\
\text { W / W \% }\end{array}$ & 4.00 & 1.10 & 7.00 & 1.20 & 6.00 & 4.00 & 3.00 & 5.60 & 1.30 \\
\hline $\begin{array}{l}\text { Potassium, } \\
\mathrm{W} / \mathrm{W} \%\end{array}$ & 2.00 & 4.00 & 7.00 & 5.00 & 3.00 & 9.00 & 1.30 & 5.00 & 4.00 \\
\hline $\begin{array}{l}\text { Iron Content, } \\
\mathrm{W} / \mathrm{W} \%\end{array}$ & 1.40 & 1.80 & 2.00 & 1.40 & 1.60 & 2.10 & 2.00 & 1.70 & 1.70 \\
\hline $\begin{array}{l}\mathrm{CCl}_{4} \\
\text { Activity }\end{array}$ & 1.72 & 1.15 & 0.75 & 1.02 & 1.19 & 1.31 & 0.32 & 1.00 & 1.35 \\
\hline $\begin{array}{l}\text { Phenol } \\
\text { adsorption } \\
\text { capacity } \\
\mathrm{mg} / \mathrm{g}\end{array}$ & 2.00 & 1.10 & 0.80 & 0.70 & 1.00 & 1.30 & 0.70 & 0.80 & 1.20 \\
\hline Yield, \% & 40 & 42 & 33 & 38 & 33 & 48 & 39 & 45 & 41 \\
\hline
\end{tabular}


Table 4 Ferronia seed shell waste activated carbon properties.

\begin{tabular}{|c|c|c|c|c|c|c|c|c|c|}
\hline Properties & $\mathrm{HCl}$ & $\mathrm{H}_{2} \mathrm{SO}_{4}$ & $\mathrm{ZnCl}_{2}$ & $\mathrm{Na}_{2} \mathrm{SO}_{4}$ & $\mathrm{Na}_{2} \mathrm{CO}_{3}$ & $\mathrm{CaCO}_{3}$ & $\mathrm{CaCl}_{2}$ & $\begin{array}{l}\mathrm{H}_{2} \mathrm{SO}_{4}+ \\
\mathrm{NH}_{4} \mathrm{~S}_{2} \mathrm{O}_{8} \\
\end{array}$ & $\begin{array}{r}\mathrm{H}_{2} \mathrm{SO}_{4} \\
+\mathrm{H}_{2} \mathrm{O}_{2} \\
\end{array}$ \\
\hline $\mathrm{pH}$ & 6.33 & 5.32 & 6.52 & 9.74 & 9.11 & 8.72 & 8.34 & 7.14 & 6.94 \\
\hline $\begin{array}{l}\text { Moisture } \\
\text { content, \% }\end{array}$ & 8.00 & 8.00 & 6.00 & 6.00 & 6.00 & 10.10 & 5.60 & 7.90 & 10.10 \\
\hline $\begin{array}{l}\text { Ash content, } \\
\%\end{array}$ & 4.35 & 10.87 & 16.38 & 12.28 & 14.89 & 8.89 & 9.79 & 28.70 & 11.11 \\
\hline $\begin{array}{l}\text { Volatile } \\
\text { matter \% }\end{array}$ & 9.80 & 12.20 & 13.00 & 11.80 & 12.00 & 10.80 & 11.40 & 11.00 & 9.80 \\
\hline $\begin{array}{l}\text { Fixed } \\
\text { carbon, \% }\end{array}$ & 82.20 & 79.80 & 81.00 & 82.20 & 82.00 & 79.10 & 83.00 & 81.10 & 80.10 \\
\hline $\begin{array}{l}\text { Conductivity } \\
\mathrm{mS} / \mathrm{cm}\end{array}$ & 1.98 & 4.10 & 0.90 & 1.13 & 1.69 & 0.45 & 0.67 & 0.77 & 0.20 \\
\hline $\begin{array}{l}\text { Specific } \\
\text { gravity, S }\end{array}$ & 1.03 & 1.25 & 0.86 & 0.63 & 0.83 & 0.63 & 0.82 & 1.25 & 0.81 \\
\hline $\begin{array}{l}\text { Bulk } \\
\text { density, D }\end{array}$ & 0.46 & 0.75 & 0.36 & 0.35 & 0.42 & 0.28 & 0.32 & 0.70 & 0.48 \\
\hline $\begin{array}{l}\text { Porosity } \\
\text { (S-D/S) } \\
\text { X100, \% }\end{array}$ & 55.34 & 40.00 & 58.14 & 44.44 & 49.40 & 55.56 & 60.98 & 44.00 & 40.74 \\
\hline $\begin{array}{l}\text { Matter } \\
\text { soluble in } \\
\text { water, \% }\end{array}$ & 0.89 & 0.41 & 0.69 & 1.15 & 1.34 & 0.96 & 0.52 & 1.06 & 0.62 \\
\hline $\begin{array}{l}\text { Matter } \\
\text { soluble in } \\
\text { Acid, \% }\end{array}$ & 0.80 & 0.40 & 0.65 & 1.55 & 1.57 & 1.43 & 0.65 & 1.32 & 0.77 \\
\hline $\begin{array}{l}\text { Surface } \\
\text { area, } \mathrm{m}^{2} / \mathrm{g}\end{array}$ & 931 & 574 & 632 & 421 & 489 & 283 & 792 & 511 & 589 \\
\hline $\begin{array}{l}\text { Iodine } \\
\text { number, } \\
\mathrm{mg} / \mathrm{g}\end{array}$ & 890 & 545 & 610 & 405 & 472 & 270 & 755 & 482 & 542 \\
\hline $\begin{array}{l}\text { Sodium } \\
\text { W / W \% }\end{array}$ & 8.00 & 1.20 & 1.80 & 13.00 & 4.00 & 8.00 & 2.50 & 5.00 & 6.00 \\
\hline $\begin{array}{l}\text { Potassium, } \\
\text { W / W \% }\end{array}$ & 1.30 & 1.80 & 5.40 & 11.50 & 1.00 & 4.00 & 10.00 & 3.00 & 4.70 \\
\hline $\begin{array}{l}\text { Iron Content, } \\
\mathrm{W} / \mathrm{W} \%\end{array}$ & 1.20 & 1.00 & 1.20 & 0.70 & 1.70 & 1.00 & 1.20 & 1.40 & 0.80 \\
\hline $\begin{array}{l}\mathrm{CCl}_{4} \\
\text { Activity }\end{array}$ & 1.14 & 2.15 & 0.42 & 0.90 & 0.72 & 1.42 & 2.30 & 0.71 & 1.72 \\
\hline $\begin{array}{l}\text { Phenol } \\
\text { adsorption } \\
\text { capacity } \\
\mathrm{mg} / \mathrm{g}\end{array}$ & 3.30 & 1.20 & 1.90 & 1.00 & 0.80 & 1.40 & 3.00 & 1.00 & 2.00 \\
\hline Yield, \% & 51 & 54 & 33 & 30 & 39 & 38 & 40 & 44 & 47 \\
\hline
\end{tabular}


Table 5. Ipomea carnia stem waste activated carbon properties

\begin{tabular}{|c|c|c|c|c|c|c|c|c|c|}
\hline Properties & $\mathrm{HCl}$ & $\mathrm{H}_{2} \mathrm{SO}_{4}$ & $\mathrm{ZnCl}_{2}$ & $\mathrm{Na}_{2} \mathrm{SO}_{4}$ & $\mathrm{Na}_{2} \mathrm{CO}_{3}$ & $\mathrm{CaCO}_{3}$ & $\mathrm{CaCl}_{2}$ & $\begin{array}{l}\mathrm{H}_{2} \mathrm{SO}_{4}+ \\
\mathrm{NH}_{4} \mathrm{~S}_{2} \mathrm{O}_{8} \\
\end{array}$ & $\begin{array}{r}\mathrm{H}_{2} \mathrm{SO}_{4} \\
+\mathrm{H}_{2} \mathrm{O}_{2} \\
\end{array}$ \\
\hline $\mathrm{pH}$ & 6.62 & 5.12 & 6.94 & 9.80 & 8.32 & 9.81 & 8.05 & 7.38 & 6.25 \\
\hline $\begin{array}{l}\text { Moisture } \\
\text { content, \% }\end{array}$ & 4.20 & 8.40 & 8.80 & 6.60 & 8.60 & 9.60 & 7.00 & 8.40 & 4.60 \\
\hline $\begin{array}{l}\text { Ash content, } \\
\%\end{array}$ & 17.75 & 10.74 & 12.06 & 7.78 & 10.64 & 18.81 & 19.68 & 27.29 & 9.43 \\
\hline $\begin{array}{l}\text { Volatile } \\
\text { matter \% }\end{array}$ & 8.50 & 9.20 & 18.80 & 14.80 & 11.20 & 17.00 & 12.00 & 10.10 & 8.80 \\
\hline $\begin{array}{l}\text { Fixed } \\
\text { carbon, \% }\end{array}$ & 87.30 & 82.40 & 72.40 & 78.60 & 80.20 & 73.40 & 81.00 & 81.50 & 86.60 \\
\hline $\begin{array}{l}\text { Conductivity } \\
\mathrm{mS} / \mathrm{cm}\end{array}$ & 0.25 & 0.26 & 0.33 & 0.41 & 0.39 & 0.10 & 0.26 & 0.30 & 0.85 \\
\hline $\begin{array}{l}\text { Specific } \\
\text { gravity, } S\end{array}$ & 0.55 & 0.83 & 0.63 & 0.92 & 0.83 & 0.93 & 0.50 & 0.83 & 1.25 \\
\hline $\begin{array}{l}\text { Bulk } \\
\text { density, D }\end{array}$ & 0.33 & 0.40 & 0.32 & 0.37 & 0.39 & 0.39 & 0.34 & 0.38 & 0.43 \\
\hline $\begin{array}{l}\text { Porosity } \\
\text { (S-D/S) } \\
\text { X100, \% }\end{array}$ & 40.00 & 51.81 & 49.21 & 59.78 & 53.01 & 58.06 & 32.00 & 54.22 & 65.60 \\
\hline $\begin{array}{l}\text { Matter } \\
\text { soluble in } \\
\text { water, \% }\end{array}$ & 0.51 & 0.35 & 0.44 & 0.59 & 0.87 & 0.84 & 0.86 & 0.35 & 0.20 \\
\hline $\begin{array}{l}\text { Matter } \\
\text { soluble in } \\
\text { Acid, \% }\end{array}$ & 0.25 & 0.49 & 1.65 & 1.07 & 1.53 & 0.88 & 0.64 & 0.31 & 0.64 \\
\hline $\begin{array}{l}\text { Surface } \\
\text { area, } \mathrm{m}^{2} / \mathrm{g}\end{array}$ & 281 & 544 & 200 & 698 & 607 & 746 & 240 & 320 & 500 \\
\hline $\begin{array}{l}\text { Iodine } \\
\text { number, } \\
\mathrm{mg} / \mathrm{g}\end{array}$ & 268 & 521 & 199 & 655 & 571 & 728 & 231 & 311 & 480 \\
\hline $\begin{array}{l}\text { Sodium } \\
\text { W / W \% }\end{array}$ & 8.20 & 1.30 & 6.00 & 13.00 & 18.00 & 1.00 & 1.20 & 3.00 & 8.50 \\
\hline $\begin{array}{l}\text { Potassium, } \\
\mathrm{W} / \mathrm{W} \%\end{array}$ & 1.20 & 1.40 & 1.90 & 3.70 & 1.30 & 1.30 & 9.00 & 1.80 & 6.00 \\
\hline $\begin{array}{l}\text { Iron Content, } \\
\mathrm{W} / \mathrm{W} \%\end{array}$ & Nil & Nil & Nil & Nil & Nil & Nil & Nil & Nil & Nil \\
\hline $\begin{array}{l}\mathrm{CCl}_{4} \\
\text { Activity }\end{array}$ & 1.21 & 1.05 & 0.72 & 0.78 & 0.52 & 0.81 & 1.32 & 1.00 & 1.15 \\
\hline $\begin{array}{l}\text { Phenol } \\
\text { adsorption } \\
\text { capacity } \\
\mathrm{mg} / \mathrm{g}\end{array}$ & 0.80 & 3.00 & 1.20 & 3.00 & 2.60 & 3.80 & 1.10 & 1.00 & 1.90 \\
\hline Yield, \% & 59 & 68 & 55 & 42 & 47 & 55 & 39 & 62 & 58 \\
\hline
\end{tabular}


Ash content value of the carbon prepared by $\mathrm{ZnCl}_{2}$ process found to be far lesser when compared to other process. The role of $\mathrm{ZnCl}_{2}$ on the removal of inorganic contents was found to be excellent. At the same time the removal of inorganic content by $\mathrm{CaCl}_{2}$ process is not an effective one. Also carbonate process and Dolomite process gives poor result on the removal of inorganic contents. The carbons prepared by other process shows comparable results with respect to ash.

Even though the role $\mathrm{ZnCl}_{2}$ on the removal of inorganic content is good, its role on the removal of volatile matter is very poor. Due to high volatile matter content the percentage of fixed carbon found low in $\mathrm{ZnCl}_{2}$ process. Optimum volatile matter and Ash content give high percentage of fixed carbon by $\mathrm{H}_{2} \mathrm{SO}_{4}+\mathrm{NH}_{4} \mathrm{~S}_{2} \mathrm{O}_{8}$ process.

Presence of water soluble impurities in the carbon prepared by Dolomite process is mainly due to the incorporation of carbonate salts in the carbon structure. Carbon prepared by all other processes exhibits moderate level of impurities. Acid soluble mettle also shows the same trend in Dolomite process, other carbons have comparable quantity of acid soluble matter.

Carbons derived in acid process are acidic in nature and carbons prepared from carbonate and sulphate process were basic in nature. No marginal variation was noticed in the conductivity values of carbons prepared in all the processes. This may be due to the presence of uniform exchangeable site on the porous surface of the activated carbon.

The iron content is low and comparable in all carbons prepared by different methods. The carbons prepared using delonix shell waste has higher level of sodium and potassium. Higher level of sodium and potassium makes the purpose of ion exchange capacity of carbon is more during the treatment of effluents.

The order of surface area of carbons prepared with different process given as follows.

$$
\mathrm{HCl}>\mathrm{CaCO}_{3}>\mathrm{Na}_{2} \mathrm{SO}_{4}>\mathrm{Na}_{2} \mathrm{CO}_{3}>\mathrm{H}_{2} \mathrm{SO}_{4}+\mathrm{H}_{2} \mathrm{O}_{2}>\mathrm{H}_{2} \mathrm{SO}_{4}>\mathrm{ZnCl}_{2}>\mathrm{H}_{2} \mathrm{SO}_{4}+
$$
$\mathrm{NH}_{4} \mathrm{~S}_{2} \mathrm{O}_{8}>\mathrm{CaCl}_{2}$

Carbon prepared using $\mathrm{HCl}$ process has high surface area. This might have been attributed to active chloride sites in the porous parts of carbon. Except $\mathrm{HCl}$ and dolomite process surface area all other carbons were found in the lower margin only. The higher value of $\mathrm{CCl}_{4}$ activity and phenol adsorption capacity confirms the surface area values. Likewise other carbons better yield obtained in dolomite and $\mathrm{H}_{2} \mathrm{SO}_{4}+\mathrm{NH}_{4} \mathrm{~S}_{2} \mathrm{O}_{8}$ and followed by acid process.

\section{Ferronia Seed Shell Waste Activated Carbon.}

Ferronia shell itself is a hard material, after carbonization it yields the quantity of fixed carbon on higher side. The characteristics of carbon indicated that the carbon obtained by all processes shows bulk density on higher side. Irrespective of treatment processes bulk density of all carbons found close to unity which makes the carbon more dense and good for treatment process.

From Table 4 it is clear that the moisture content of all carbons was found to be comparable. No remarkable difference observed in any one of the process. $\mathrm{HCl}$ and dolomite process gives encouraging results with respect to the removal of inorganic matter. Poor ability of $\mathrm{NH}_{4} \mathrm{~S}_{2} \mathrm{O}_{8}$ to effectively remove inorganic contents leads to higher value of ash content. No major differences were observed in volatile matter content in all the nine processes. Only the ash content plays a major role in deriving the amount of fixed carbon. Because of comparable amount of ash and volatile content percentage of fixed carbon found to be more or less same in all the process of carbonization. 
Table 6. List of various activation process and abbreviation of carbon

\begin{tabular}{cll}
\hline S.No & Sample & \multicolumn{1}{c}{ Treatment } \\
\hline 1. ACX1 & Acid process using $\mathrm{HCl}$. \\
2. ACX2 & Acid process using $\mathrm{H}_{2} \mathrm{SO}_{4}$ \\
3. ACX3 & Chloride process using $-\mathrm{Zn} \mathrm{Cl}_{2}$ impregnation \\
4. ACX4 & Sulphate process using $\mathrm{Na}_{2} \mathrm{SO}_{4}$ impregnation. \\
5. ACX5 & Carbonate process using $\mathrm{Na}_{2} \mathrm{CO}_{3}$ impregnation. \\
6. ACX6 & Dolomite process using $\mathrm{Ca} \mathrm{CO}_{3}$ \\
7. ACX7 & Chloride process using $-\mathrm{CaCl}_{2}$ impregnation \\
8. ACX8 & Oxidation using $\mathrm{Am}$. Persulphate $+\mathrm{H}_{2} \mathrm{SO}_{4}$ \\
9. ACX9 & Oxidation using $\mathrm{H}_{2} \mathrm{O}_{2}+\mathrm{H}_{2} \mathrm{SO}_{4}$ \\
\hline
\end{tabular}

$\mathrm{X}=\mathrm{T}$ Turmeric Industrial waste; $\mathrm{X}=\mathrm{J}$ Jatropha curcus seed shell waste; $\mathrm{X}=\mathrm{D}$ Delonix regia seed shell waste; $\mathrm{X}=\mathrm{F}$ Ferronia seed shell waste; $\mathrm{X}=\mathrm{I}$ Ipomea carnia stem waste

Table 7. Selected dyes and their characteristics taken from colour index.

\begin{tabular}{|c|c|c|c|c|c|c|}
\hline $\begin{array}{l}\text { S. } \\
\text { No }\end{array}$ & Class & Commercial Name & C.I. No & $\begin{array}{c}\text { Structure } \\
\text { / Class }\end{array}$ & $\begin{array}{c}\lambda \max \\
\mathrm{nm}\end{array}$ & $\begin{array}{c}\text { Formula } \\
\text { Weight }\end{array}$ \\
\hline 1 & Reactive & Cibacran brilliant yellow $3 \mathrm{G}-\mathrm{R}$ & 18972 & Anionic & 404.00 & 872.97 \\
\hline 2 & Direct & Direct Orange 31 & 23655 & Anionic & 670.62 & 428.00 \\
\hline 3 & Acid & Acid Blue - 40 & 62125 & Anionic & 610.00 & 473.44 \\
\hline 4 & Basic & Rhoamine B & 45170 & Cationic & 544.00 & 442.56 \\
\hline
\end{tabular}

Water soluble matter was found high only in the case of carbon prepared in $\mathrm{Na}_{2} \mathrm{SO}_{4}$ process. Remaining carbons show only a marginal variation in the water soluble matter content. This depicts that presence of water soluble impurities will not give much impact on the treated effluent water.

Acid soluble matter content was found slightly high in the carbons prepared by $\mathrm{Na}_{2} \mathrm{SO}_{4}$ and $\mathrm{Na}_{2} \mathrm{CO}_{3}$ processes. High acid soluble matter in dolomite process may be mainly because of incorporated carbonate groups in the pores.

Acidity and basicity were found to be followed the same trend as in the previous discussions. Conductivity of $\mathrm{NH}_{4} \mathrm{~S}_{2} \mathrm{O}_{8}$ carbon was found close to zero indicates the absence of charged ions inside the porous structure. Sodium and potassium content was found to high in $\mathrm{Na}_{2} \mathrm{SO}_{4}$ carbon, high sodium content may be due to incorporation of medium ion present in the treatment solution and potassium content can be brought from the mineral storage in shells. Iron content is slightly high is all the processes, which may affect during effluent treatment process by leaching.

Surface area of carbons prepared in different process given in the following order. $\mathrm{HCl}>\mathrm{CaCl}_{2}>\mathrm{ZnCl}_{2}>\mathrm{H}_{2} \mathrm{SO}_{4}+\mathrm{H}_{2} \mathrm{O}_{2}>\mathrm{H}_{2} \mathrm{SO}_{4}>\mathrm{H}_{2} \mathrm{SO}_{4}+\mathrm{NH}_{4} \mathrm{~S}_{2} \mathrm{O}_{8}>\mathrm{Na}_{2} \mathrm{CO}_{3}>\mathrm{Na}_{2} \mathrm{SO}_{4}>$ Dolomite

Out of nine carbons prepared using ferronia shell, carbon prepared using $\mathrm{CaCl}_{2}$ process shows an excellent action on making high porosity through proper removal volatile, organic and inorganic contents. Surface area data were again conformed with the help of $\mathrm{CCl}_{4}$ activity and phenol adsorption capacity data. Unlike earlier carbons dolomite process gives only an average yield and the acid processes conforms their contribution towards high yield. 


\section{Ipomea Carnia Stem Waste Activated Carbon}

From Table 5 it is observed that bulk density of carbons prepared from different process show a comparable value. No major deviations were noticed in bulk density. This uniform bulk density implies that Ipomea stem is highly resistant towards chemicals and the pore system is also uniform irrespective of the processing methods.

The slightly higher moisture content in the carbon prepared by dolomite process may be due to moisture adsorptive nature of carbonate groups present in the activated pores of carbon particle ${ }^{26}$. On comparing the carbons prepared by various method, the carbon prepared by $\mathrm{H}_{2} \mathrm{SO}_{4}+\mathrm{NH}_{4} \mathrm{~S}_{2} \mathrm{O}_{8}$ process has high ash content, this might be due to its poor ability to remove inorganic contents present is carbon. In this task $\mathrm{Na}_{2} \mathrm{SO}_{4}$ and $\mathrm{H}_{2} \mathrm{SO}_{4}+\mathrm{H}_{2} \mathrm{O}_{2}$ process gives good results is removing the inorganic contents. The ash content values from Table 5 indicated that the overall ash content for all the varieties of carbon prepared shows around $15 \%$ attributed to moderate inorganic content and high fixed carbon.

The results of solubility studies in water and acid show that water soluble matter content is high in $\mathrm{Na}_{2} \mathrm{CO}_{3}$ and $\mathrm{CaCl}_{2}$ processes. The acid leachable matter was also found to be high in the carbon prepared by the $\mathrm{ZnCl}_{2}$ process.

Except the carbons prepared by acid process, all the other carbons show alkaline nature. The high $\mathrm{pH}$ (i.e. alkalinity) can be related to the introduction of some basic groups and removal of acidic functional groups with other processes compared to acid process. Conductivity values do not shows much variation, this means that cations in acidic carbons and anions in basic carbons may be responsible for the conductivity.

The iron content is below the detectable limit in all the carbons prepared by different process. This implies that the carbon prepared using stem components can be utilized in the treatment of effluent water without the problem of iron leaching. The level of sodium is slightly higher than potassium. This sodium and potassium content may be derived from the ionic transport in the stem during nutrient transfer.

The surface area of carbons prepared in different process is given as follows.

$$
\begin{aligned}
& \text { Dolomite }>\mathrm{Na}_{2} \mathrm{SO}_{4}>\mathrm{Na}_{2} \mathrm{CO}_{3}>\mathrm{H}_{2} \mathrm{SO}_{4}>\mathrm{H}_{2} \mathrm{SO}_{4}+\mathrm{H}_{2} \mathrm{O}_{2}>\mathrm{H}_{2} \mathrm{SO}_{4}+\mathrm{NH}_{4} \mathrm{~S}_{2} \mathrm{O}_{8}> \\
& \mathrm{ZnCl}_{2}
\end{aligned}
$$

The higher surface area of carbon prepared by Dolomite process is attributed to the effective removal of organic contents by $\mathrm{CO}_{2}$ produced by Dolomite process. The surface area of activated carbon by dolomite process is comparable with other activated carbons produced from Agricultural wastes. The phenol adsorption capacity and $\mathrm{CCl}_{4}$ activity varies with surface area. The high adsorption capacity of Dolomite process carbon conforms its high surface area. Alkaline nature of Dolomite process carbon is desirable property for the effective removal of acidic dyes is aqueous solution. From Table 5 the values of yield indicated that like in turmeric waste, the acid process produces more yield when compared to other process.

\section{Comparative Study on Characteristics of Carbon}

It is observed that in all processes of turmeric waste carbon and ipomea stem waste carbon gives higher yield than other varieties of carbons. Bulk density of carbons obtained from all the materials shows that ferronia seed shell waste carbon has the higher bulk density and ipomea stem carbon has the lower bulk density, which can be attributed to the material hardness. 
Ash content generally gives an idea about inorganic constituents associated with carbon $^{26}$. The data indicate that the ash content for all the varieties of carbons are very low thereby increasing the fixed carbon content except for the carbon obtained from ipomea carnia and ferronia seed shell waste carbon by $\mathrm{H}_{2} \mathrm{SO}_{4}+\mathrm{NH}_{4} \mathrm{~S}_{2} \mathrm{O}_{8}$.

The data pertaining to mater soluble in acid and indicate that except carbons prepared by acid process carbon obtained from all other process exhibit small amount of leaching property. The low percentage of soluble matter in the case of carbons obtained by acid process is known that during the preparative stage itself, the soluble matters are leached out by acid from the carbon skeleton.

In general the carbons derived from acid process shows acidic properties and all the remaining carbons exhibit neutral and basic properties. This basic nature resembles with commercial activated carbon. Characterization studies on porosity, surface area. Iodine number, $\mathrm{CCl}_{4}$ activity and phenol adsorption capacity clearly indicates that the carbons obtained by various processes will depend only on the composition of raw agricultural waste. Surface area properties of $\mathrm{HCl}$ process for ferronia carbon, $\mathrm{HCl}$ and $\mathrm{CaCl}_{2}$ processes for jatropha carbon, $\mathrm{HCl}$ process for delonix carbon, chloride process for turmeric waste and Dolomite process for ipomea carbon are comparable with commercial activated carbon.

Iron content is almost uniform for all the five carbons and also it will not give any major conclusion about the nature of process. This level of iron content will not affect the effluent water without the problem of iron leaching into treated water.

Evaluation of Activated carbon samples for the removal of dyes.

In order to simplify the discussion of the adsorption experiments the results were derived in terms of five different activated carbon source viz. activated carbon prepared from 1.Turmeric industrial waste, 2.Jatropha curcus seed shell waste, 3.Delonix seed shell waste, 4.ferronia seed shell waste and 5. ipomea stem waste.

Acid, reactive and direct dyes have anionic character and basic dyes are cationic character. Figures. 1 to 5 shows the percentage of anionic and cationic dye removal for 5 different activated carbons prepared from agricultural wastes viz. turmeric industrial waste(ACT), jatropha curcus seed shell waste(ACJ), delonix seed shell waste(ACD), ferronia seed shell waste(ACF) and ipomea stem waste(ACI) respectively.

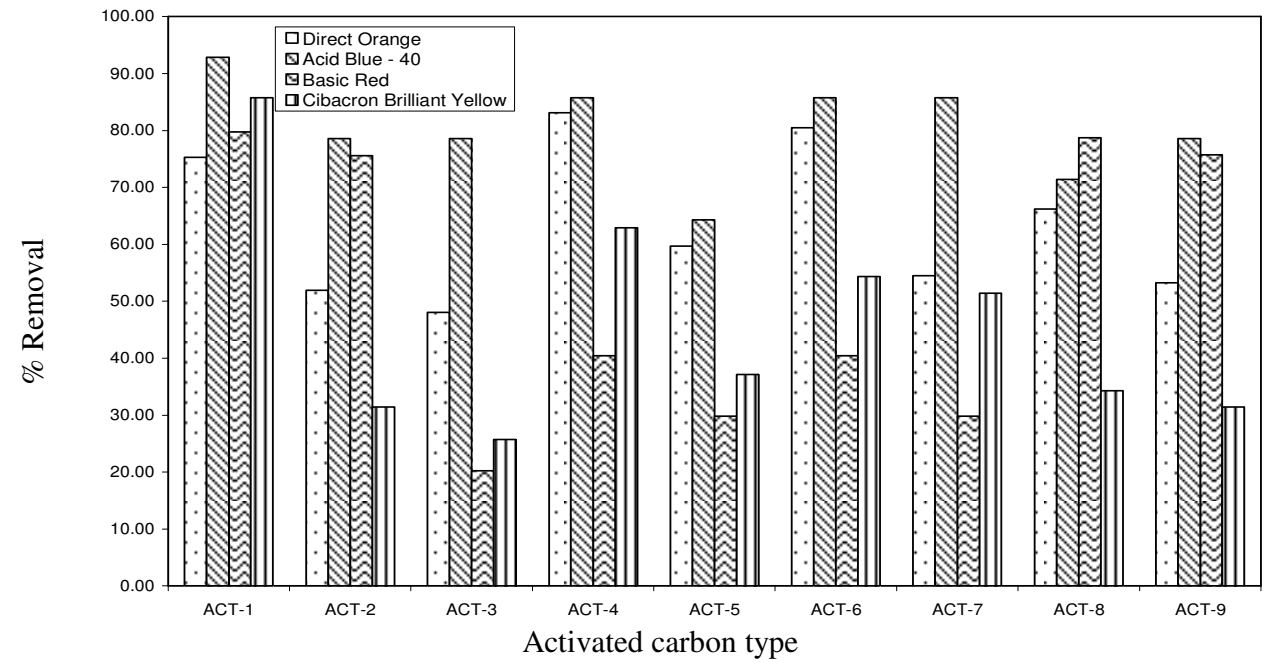

Figure 1. Percentage of dye removal by activated carbon ACT 


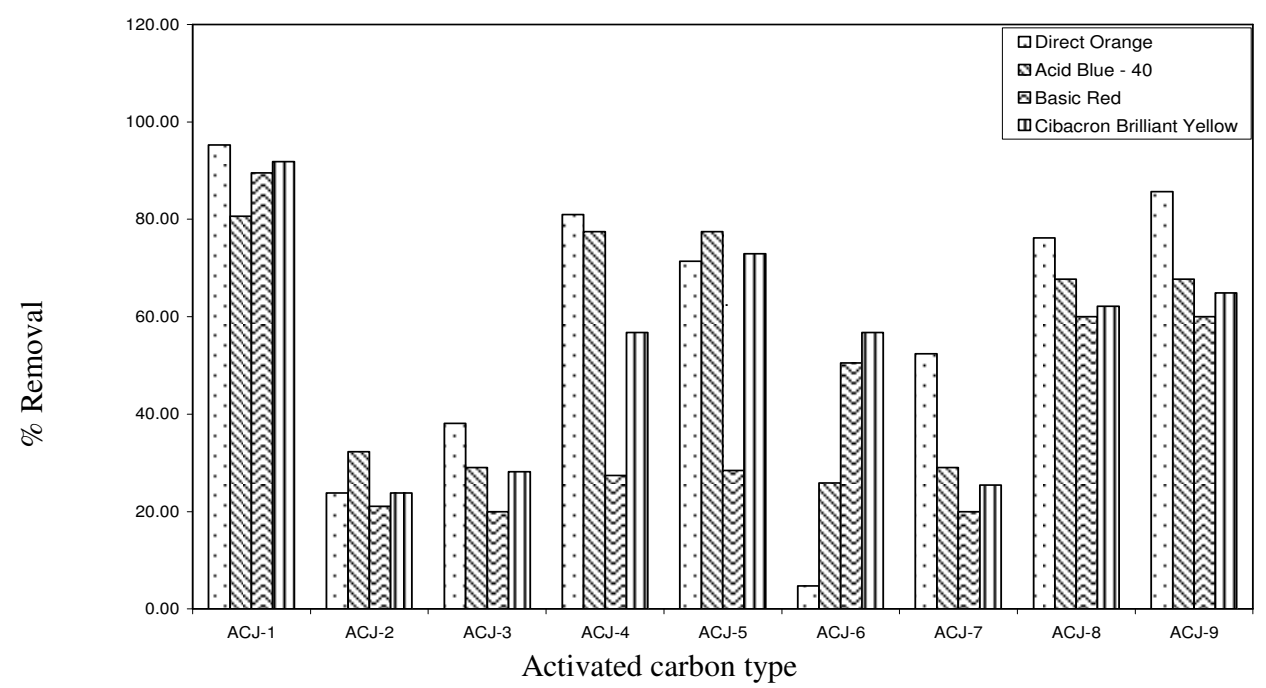

Figure 2. Percentage of dye removal by activated carbon ACJ

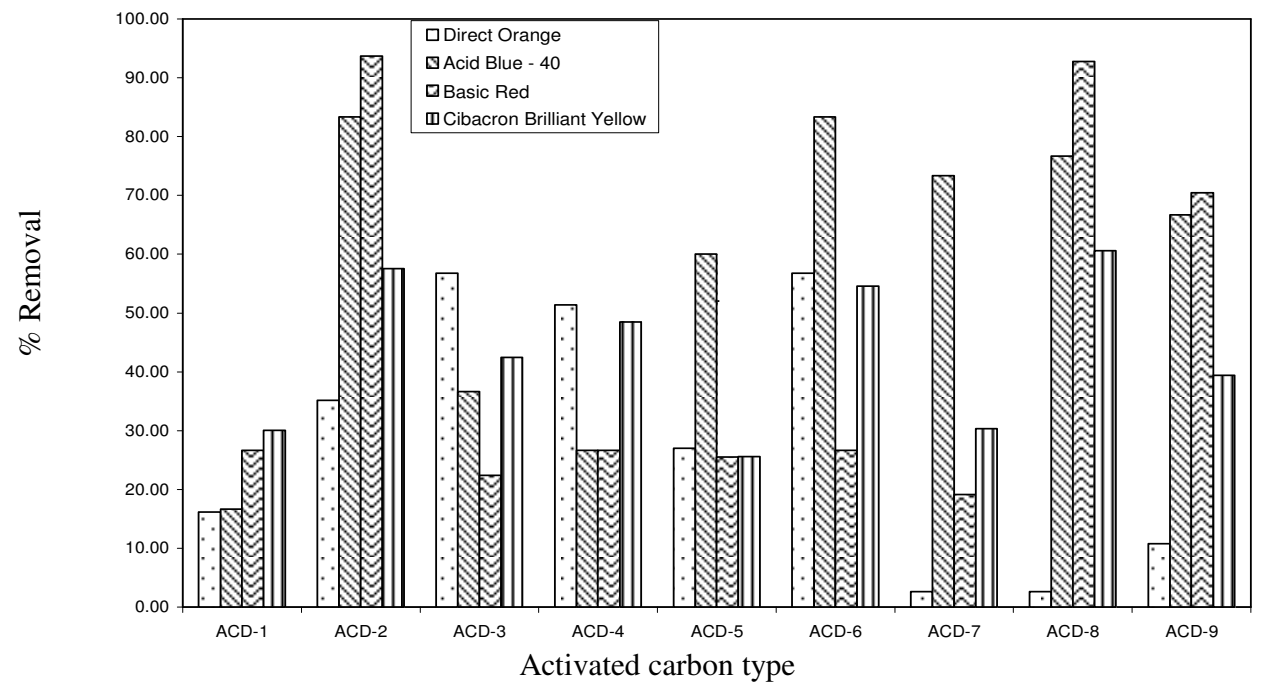

Figure 3. Percentage of dye removal by activated carbon ACD

A close relationship between surface area and surface groups of the modified activated carbons and the percentage of dye removal by adsorption can be observed.

For the nine types of activated carbons prepared from turmeric industrial waste carbon, a similar behavior was observed for anionic dyes (i.e acid, direct and reactive dyes) following an increase in the adsorption capacity with increase in the surface area. Different performances are obtained for the materials tested, varying from $25.75 \%$ ( for ACT3 ) to $92.90 \%$ (for sample ACT1 ) of anionic dye removal for the turmeric waste activated carbon. sample ACT1 is the most efficient material for the adsorption of all the anionic dyes tested.

This difference in the uptake of anionic dyes cannot be explained on the basis of surface area alone, as ACT1 which has smaller surface area than ACT3 adsorbs larger amount anionic dyes. As the carbons prepared using different preparation procedures, these carbons are expected to have different chemical structures and textural properties on their surface. 


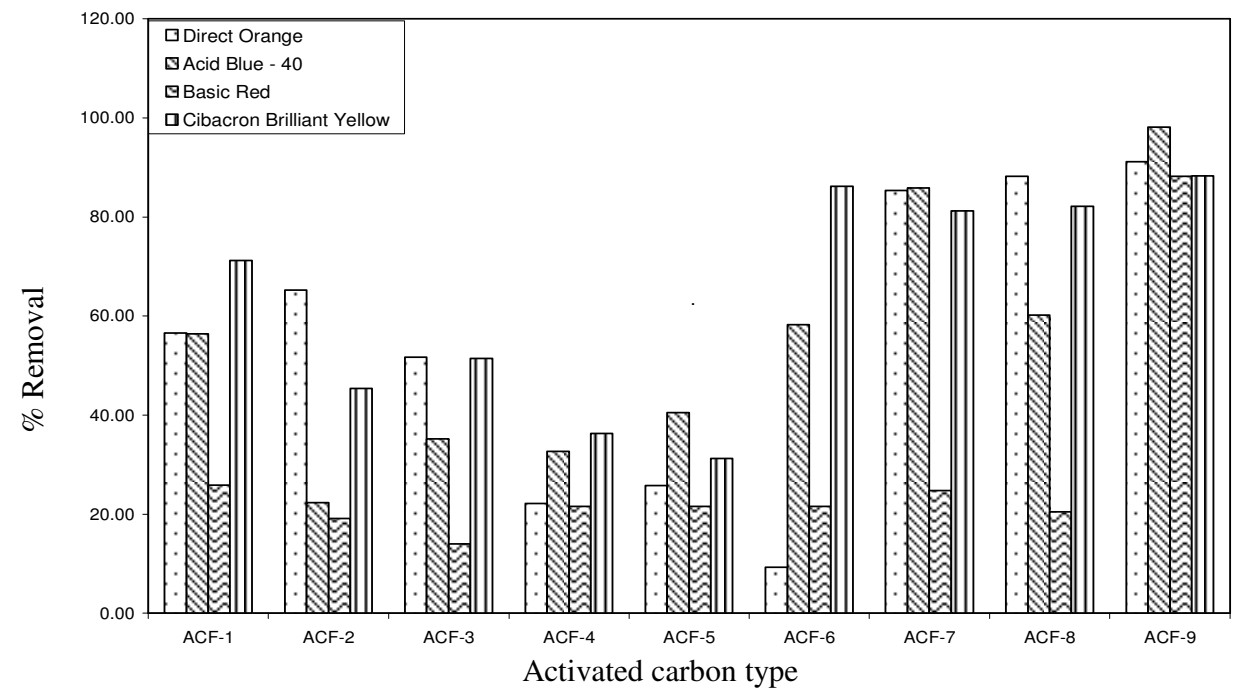

Figure 4. Percentage of dye removal by activated carbon ACF

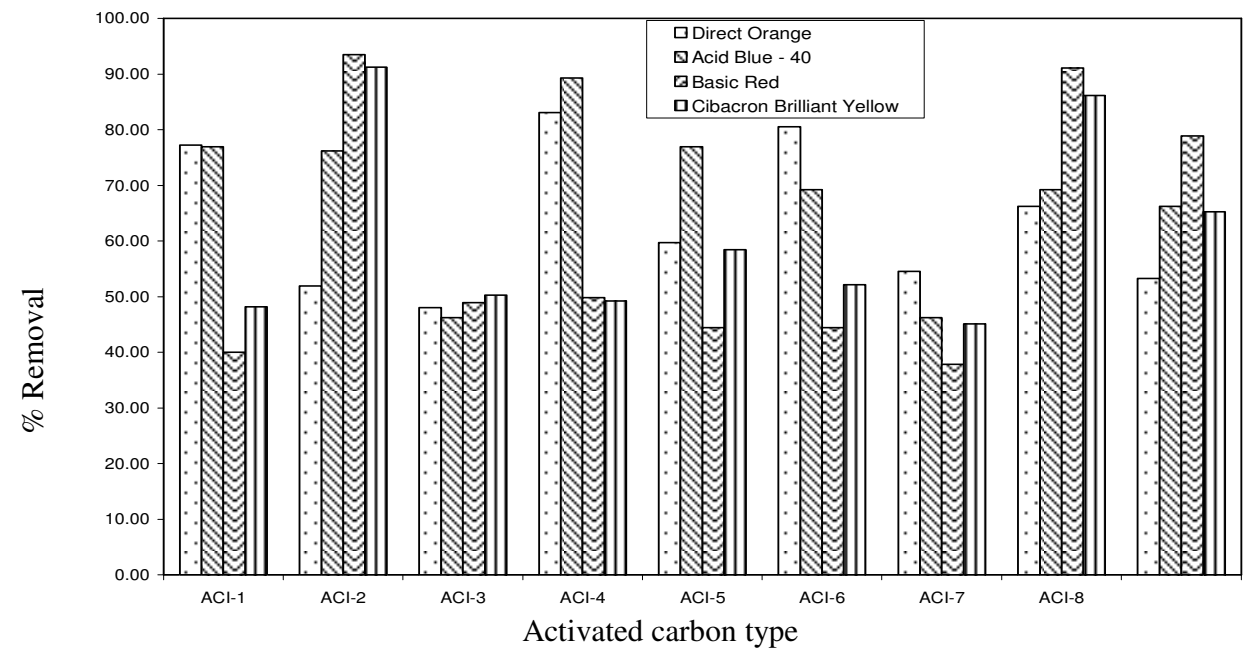

Figure 5. Percentage of dye removal by activated carbon ACI

This indicates that as shown by several earlier workers ${ }^{18,19,23}$ the surface chemical structure of the carbon also influences the dye adsorption. Basic dyes have a cationic character and different behavior was expected for their adsorption on modified activated carbons prepared from turmeric industrial waste in comparison to that of the anionic dyes. The major difference observed for basic dyes in comparison to anionic dyes is the good performance observed the acid treated samples ACT1, ACT2, ACT8 and ACT9. This was expected since the large amount of surface acid groups presented in the sample ( mainly carboxylic, anhydride, lactones and phenols etc.) are good anchoring sites for basic dyes as a result of electronic interactions. The existence of this type of adsorption mechanism has been reported by Perira.R et $_{\text {al. }}{ }^{27}$, who observed the existence of this type of adsorption mechanism in the adsorption of basic dyes on the activated carbons.

The jatropha curcus seed shell waste activated carbon has a similar behaviour to that described for ACT carbons. In general terms ACT1 sample has the best performance and ACT3 sample the worst performance in adsorbing the anionic dyes. This behavior shows 
that the adsorption of dyes and surface mechanism involved in the textural properties of the activated carbon prepared using jatropha curcus are the same as that of ACT. Acid dyes also behave similar to reactive dyes, the ACJ1, ACJ4, ACJ5, ACJ8 and ACJ9 are being the best and $\mathrm{ACJ} 2 \& \mathrm{ACJ} 3$ the worst material for adsorbing acid dyes.

The chemical structure of carbon surface is due to the presence of associated oxygen which is present in the form of two types carbon-Oxygen surface groups; one which is evolved as $\mathrm{CO}_{2}$ and the second is evolved as $\mathrm{CO}$ on degassing ${ }^{24,25}$.

In order to examine the influence of these type of surface groups on the adsorption (Cationic and anionic dyes more clearly), activated carbons ACD, ACF and ACI were oxidized with $\mathrm{H}_{2} \mathrm{SO}_{4}$, ammonium persulphate and $\mathrm{H}_{2} \mathrm{O}_{2}$ solutions. These oxidative treatments are known to enhance the amount of these Carbon-Oxygen surface groups. The adsorption percentage of anionic and cationic dyes on these oxidized samples is presented in the Figure 3, 4 and 5 for the shake of easy comparison.

Further more the decrease in adsorption is much larger in case of samples oxidized with $\mathrm{H}_{2} \mathrm{SO}_{4}$, and much smaller when the sample is oxidized with ammonium persulphate and $\mathrm{H}_{2} \mathrm{O}_{2}$ in case of Activated carbons prepared from ACF. The existence of this type of mechanism is reported Bansal R.C et al. ${ }^{26}$ who observed the existence of these type of adsorption mechanism in the adsorption of phenol on activated carbons.

The adsorption of organic molecules by activated carbon from aqueous solutions has been studied by number of workers, but the results have been interpreted differently. Bansal R.C et $a l^{26}$ attributed adsorption of organic molecules on the activated carbon surface to charge transfer interaction between the adsorbate and the surface carbonyl groups or between the adsorbate and the fused ring system of the basal planes of the carbon.

Carbonyl oxygen groups on the carbon surface act as a electron donors and the aromatic ring of the solute as the acceptor. Graham ${ }^{20}$, Coughlin et al ${ }^{21}$ and Puri et al. ${ }^{23}$ are of the view that the presence of carbon oxygen surface groups also influences the adsorption of phenol through the formation of bond with non-acidic oxygen surface groups.

The results presented in these investigations show that the oxidation of carbon with $\mathrm{H}_{2} \mathrm{SO}_{4}$, which preferably creates sulphonic and lactonic acid groups suppress the adsorption of anionic dyes. In the case of ACF2, however when the surface groups are altered by different activation processes the adsorption of anionic and cationic dyes either tends to increase of decrease. The results of such studies are presented in the Figures 4 and 5 for the activated carbon prepared from delonix seed shell and ipomea carnia stem waste activated carbons.

\section{Conclusion}

From the results of the present investigation, it can be concluded that:

1. Activated carbons can be conveniently and economically prepared from these five agricultural wastes.

2. The extensive characterization studies of the different varieties of activated carbon reveal that the carbons obtained from all the raw materials can be assessed as superior grade carbons.

3. Surface chemical groups of activated carbon play a key role in dye adsorption along with surface area. Starting from same material and using appropriate chemical treatments to modify their surface chemistry without changing significantly their textural properties, a substantial variation in the dye removal capacity was observed. For several of the dyes tested, activated carbons with different surface characteristics can lead to differences up to 80 percentages in the amount of dye removed. 
4. Acid processes were the most efficient among the chemical treatments performed to modify the surface chemistry, Chloride process for $\mathrm{ACT}, \mathrm{HCl}$ and $\mathrm{CaCl}_{2}$ processes for $\mathrm{ACJ}$ and $\mathrm{HCl}$ process for $\mathrm{ACD}$ and $\mathrm{ACF}$ gives carbon with higher surface area.

5. For anionic dyes (reactive, direct and acid) a close relationship between the surface area and surface chemical groups of the modified activated carbon and the percentage of dye removal by adsorption can be observed.

6. Cationic dyes large amount of acid groups present in the sample (mainly carboxylic anhydrides, lactones and phenols etc.) are good anchoring sites for the adsorption.

7. Hence, the activated carbons obtained from all the five raw materials can be used for the removal of wide variety of impurities from the waste water.

\section{References}

1. Cheremisinoff N P and Morresi A C, Carbon adsorption applications, Carbon adsorption Handbook, Ann Arbor Science Pub, Inc: Ann Arbor Michigen, 1980, 1.

2. Bansal R C, Donnet J B and Stoeckli F, Active carbon, Marcel Dekker, Inc, New York, 1988.

3. Mantell C L, Carbon and Graphite Handbook. John Wiley \& Sons Inc, New York, 1968.

4. Lopez-Gonzalez D J, Adv Sci Techno, 1984,1,103.

5. Linares-Solano, Lopez-Gonzalez D J, Molina-Sabio M and Rodriguez-Reinoso F, J Chem Tech Biotechnol, 1980, 30, 65.

6. Nasser M M and El-Geundi M S, J Chem Biotechnol, 1991, 50, 257.

7. Bousher A, Shen V and Edyvean R G J, Wat Res, 1997, 31, 2084.

8. Kadirvelu K, Palanivel M, Kalpana R and Rajeshwari S, Bioresource Technol, 2000, 74, 263.

9. Srinivasan K, Balasubramanian $\mathrm{N}$ and Ramakrishna $\mathrm{T} \mathrm{V}$, Ind $J$ Environ Health, 1998, 30, 376.

10. Rengaraj S, Banumathi A and Murugesan B, Ind J Chem Technol, 1999, 6, 1.

11. Banerjee S K, Majmudar S, Roy A C, Banerjee S C and Banerjee D K, Ind J Technol, 1976, 14, 45.

12. Morais L C, Goncalves E P, Vasconcelos L T and Beca C G G, Environ Technol, 2000, 21577.

13. Balasubramainan M R and Muralisankar I, Ind J Technol, 1987, 25, 471.

14. Mittal A K and Venkobachar C, Ind J Environ Health, 1989, 31,105.

15. Lucchesi A and Maschio G, Conserv Recyc, 1983, 6, 85.

16. Lambert S D, Graham N J D, Sollars C J and Fowler G D, Wat Sci Technol, 1997, 36,173.

17. Al-Qodah Z, Wat Res, 2000, 34, 4295.

18. Xu X, Shi W and Sun G, Ind Engg Chem Res, 1997, 36, 808.

19. Reckhow D A, Singer P C and Malcolm R L, Environ Sci Technol, 1990, 24, 1655.

20. Karanfil T, Kilduff J E, Kitis M and Wigton A, Environ Sci Technol, 1999, 33, 3225.

21. Lee M C, Snoeyink V L and Crittenden J C, J Am Water Works Assoc, 1981,73, 440.

22. Summers R S and Roberts P V, J Colloid Interface Sci, 1988,122, 367.

23. Summers R S and Roberts P V, J Colloid Interface Sci, 1988, 122, 382.

24. Rengaraj S, Banumathi Arabindo and Murugesan V, J Sci Ind Res, 1998, 57,129.

25. ISI, Activated Carbon , Powdered and Grannuar - Methods of sampling and its tests, ( Bureau of Indian Standards, New Delhi), IS 877 - 1989.

26. Bansal RC, Diksha Agarwal, Meenakshi Goyal and Krshna B C, Ind J Chem Tech, 2002, 9, 290.

27. Pereira R, Samanta F Soras, Jose J M and Jose L Figueiredo, Carbon, 2003, 41, 811. 


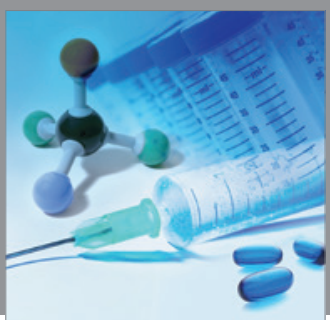

International Journal of

Medicinal Chemistry

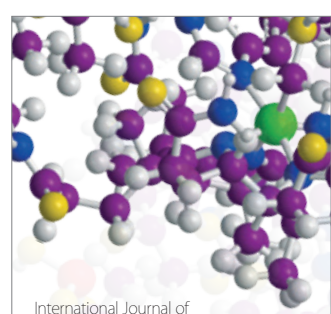

Carbohydrate Chemistry

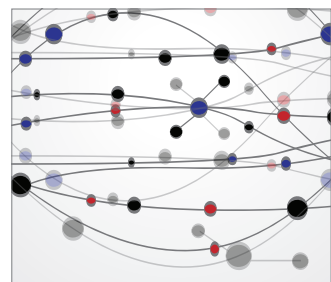

The Scientific World Journal
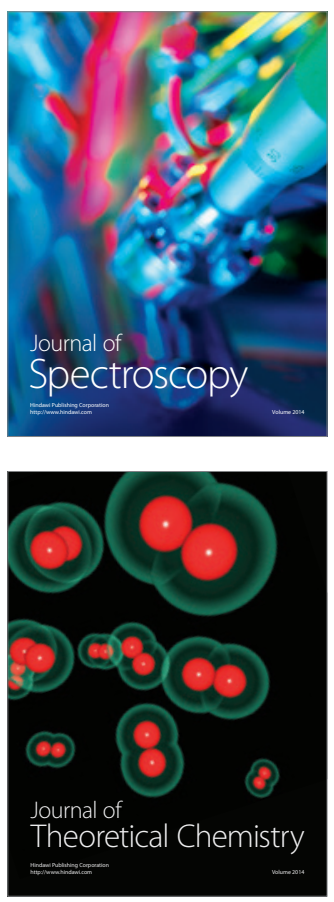
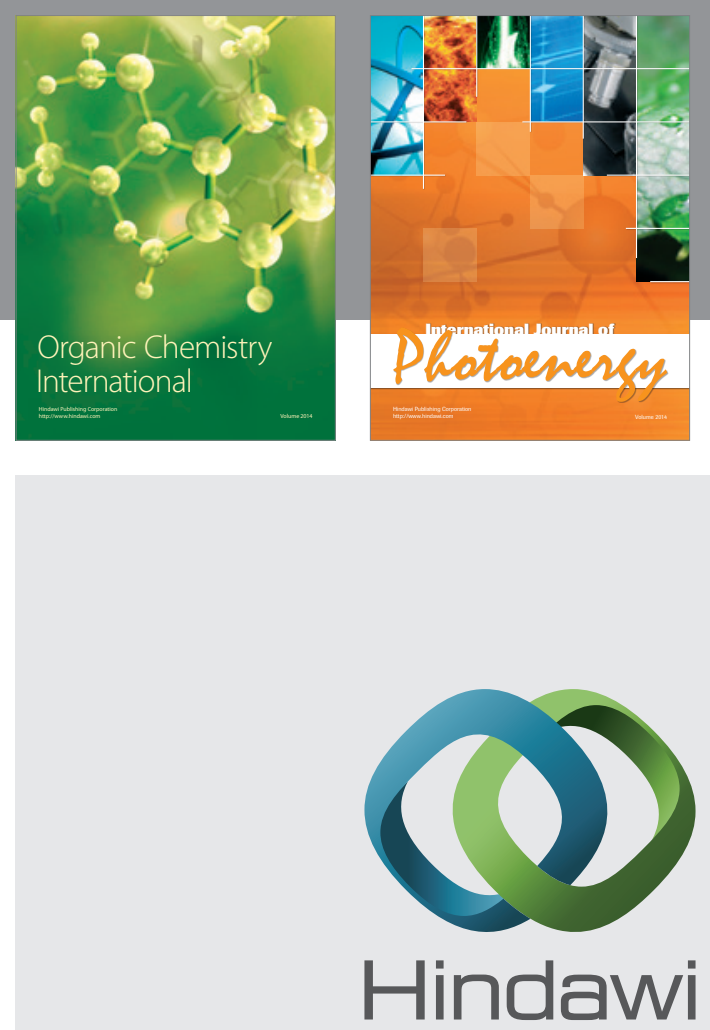

Submit your manuscripts at

http://www.hindawi.com
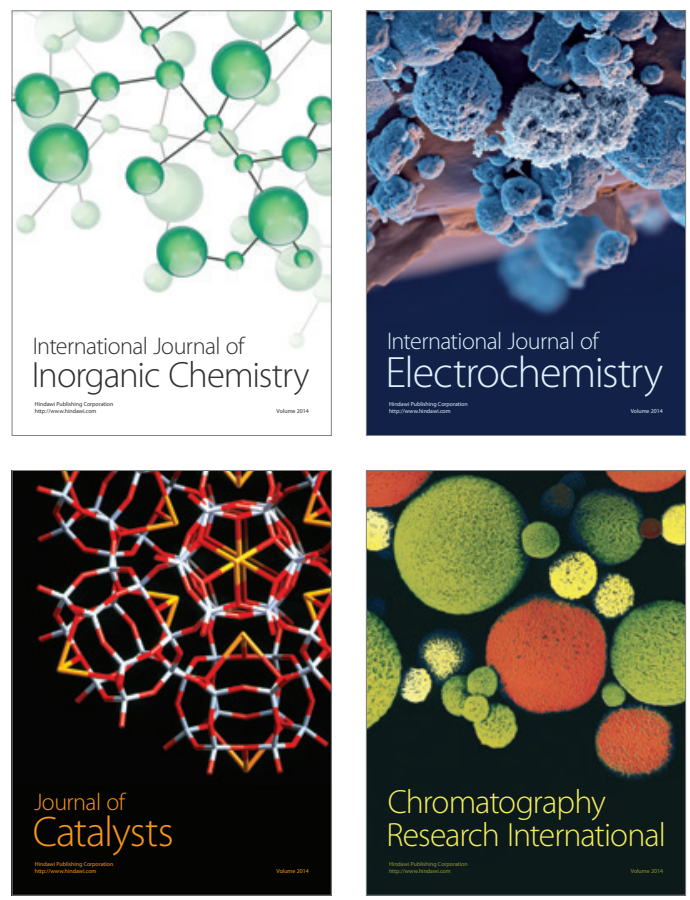
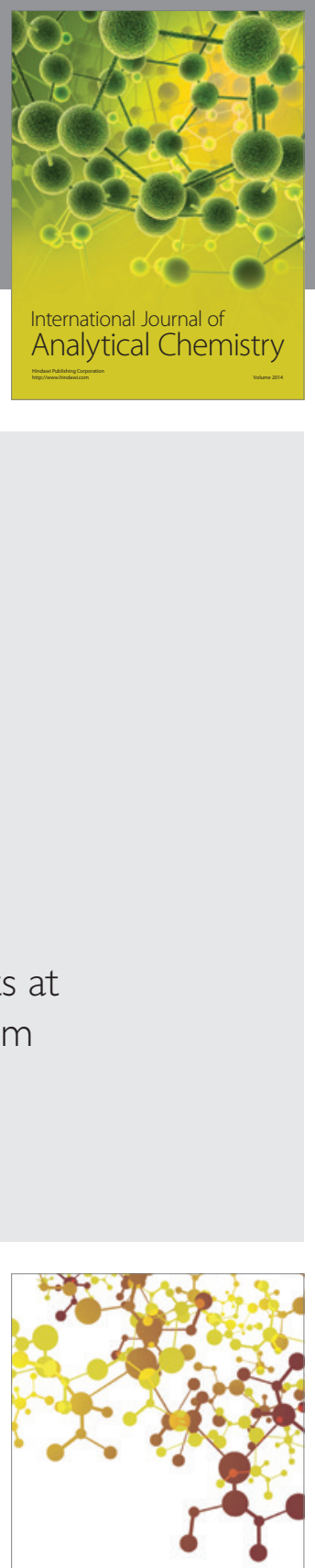

Journal of

Applied Chemistry
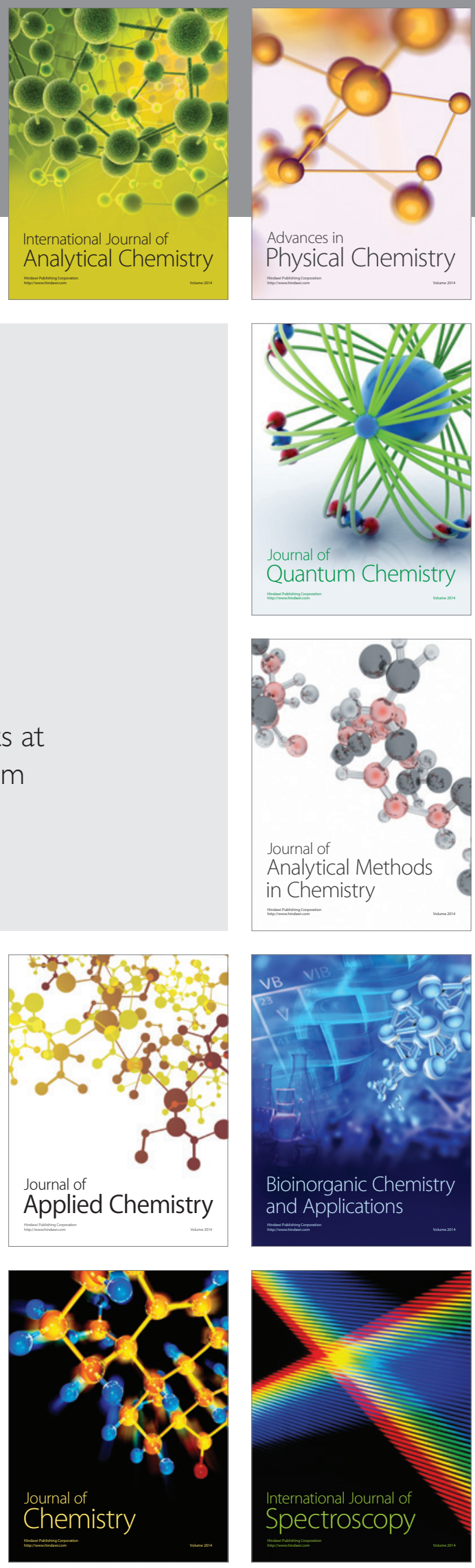